\title{
Predicting Car States through Learned Models of Vehicle Dynamics and User Behaviours
}

\author{
Theodosis Georgiou* and Yiannis Demiris*
}

\begin{abstract}
The ability to predict forthcoming car states is crucial for the development of smart assistance systems. Forthcoming car states do not only depend on vehicle dynamics but also on user behaviour. In this paper, we describe a novel prediction methodology by combining information from both sources - vehicle and user - using Gaussian Processes. We then apply this method in the context of high speed car racing. Results show that the forthcoming position and speed of the car can be predicted with low Root Mean Square Error through the trained model.
\end{abstract}

\section{INTRODUCTION}

With rapid developments in technology, advanced driving assistance systems have become increasingly widespread. These systems enhance cars for safety and comfort through the use of sensors smartly located to observe the driver, vehicle and environment. However, embraced approaches do not take the driver experience and behaviour into account. An intelligent vehicular system would have to infer the intentions of the driver and help or intervene only when needed. Having a system that is tuned to the capabilities of the driver would predict the user's behaviour and therefore assist or warn before any user action is taken. Developing a system tailored to the user generates a number of future opportunities: give assistance only when needed, create training programs tailored for particular user weaknesses, perform collision avoidance or even user identification and theft detection.

In this paper, we propose a probabilistic approach, using Gaussian Process Regression, for implementing a driver's behaviour model from which we can infer forthcoming system states. As seen in Figure 1, the user model is trained by collecting data from the user, vehicle and environment through a racing car simulator. During training, the algorithm builds up a model that describes the user's behaviour combined with the vehicle's dynamics which is then used to foresee upcoming system states. Our hypothesis is that when a particular driver comes across similar conditions, (s)he will create system states similar to his/her training data.

\section{RELATED WORK}

Researchers are attempting different techniques to teach, learn and predict the user intentions so as to improve driving assistance systems. Some try to analyse and construct a driving behaviour model [1], [2] in order to recognise and predict individual users' actions: turning left, changing lane, etc. Others try to train the driver in unexpected events [3] or

\footnotetext{
*Theodosis Georgiou and Yiannis Demiris are with the Personal Robotics Laboratory, Department of Electrical and Electronic Engineering, Imperial College London, SW7 2BT, UK \{theodosis.georgiou08, y.demiris\}@imperial.ac.uk
}

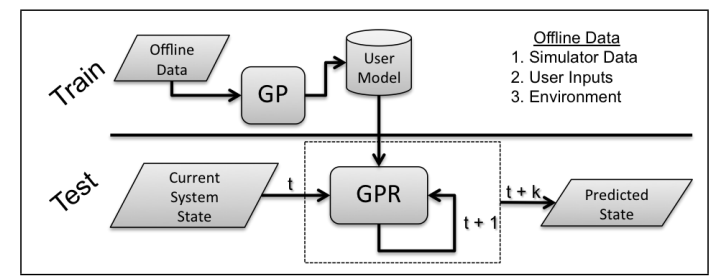

Fig. 1. The GPR Prediction Process. Gaussian Process Regression algorithm (GPR) uses the already trained user model to predict the next system state from the current state created by the user. Predicted state is pushed back into the algorithm to increase the time of forecasting.

construct a suitable training program to minimise the reaction time of the driver and thus avoid prominent collisions [4], [5].

\section{A. Driver Behaviour for Prediction and Identification}

Studies in driver behaviour and technology advancement in vehicles have been an important topic for many years [6], [7]. Related studies revealed that researchers focusing on driver behaviour are divided into two main categories: driver identification and driver action prediction. The first category concentrates on identifying individual drivers or grouping a set of drivers through a suitable set of inputs [8], [9]. Erzin et al. [10] presented a system that can detect if the driver is drunk, distracted or fatigued through the user behaviour, in order for assistive action to be taken.

In the second category, studies relating to driver action prediction are trying to abstract and model a set of driving action patterns (turn left/right, roundabout turns, changing line, following a vehicle, etc.) through inputs from different sensors (gas/brakes pedals, gaze, steering, speed, etc.), using machine learning algorithms, so as to recognise the action from incomplete set of inputs, thus recognising an action before it actually happens. The reason for predicting the action is to detect and avoid potentially dangerous situations [1], [2], [11], [12], [13], [14].

In our research, we aim to take the driver's action prediction one step further so as to estimate multiple forthcoming low level system states as shown in Table I. In this paper we are showing the prediction results and refinements from 3 variables of the state: the $2 \mathrm{D}$ position $(\mathrm{X}-\mathrm{Y})$ and speed of the vehicle by using the help of the driving history of the user and current system state, embedded in a Gaussian Process machine learning model. These variables are important to algorithms deciding whether the user will need help or not. They also provide valuable information to the driver.

\section{B. Time Series Forecasting using Gaussian Process}

Forecasting time series using Gaussian Process (GP) has been very popular in the last decade due to its good per- 
formance in modelling dynamic systems. Related to our approach, Ko et al. [15] used Gaussian Process Regression with Reinforcement Learning in order to model the dynamics of an autonomous blimp. By comparing non-linear dynamic models of a blimp and enhanced Dynamics models with GP models, they showed that the predictions with GPs were more accurate since the GP models capture information that cannot be deduced by their non-linear models. The results showed that the RMS errors of their GP prediction outputs were on average 5 times lower than those of the non-linear models.

For a time series of length $\mathrm{t}$, the prediction of a forthcoming state at time $t+k$ can be either done iteratively or directly. Iterative approach is when the prediction of each step is used to predict the immediate next step until we reach the desired state. The direct approach steps ahead to the prediction of that state but requires retraining the model if $k$ changes. It has been shown that the direct method is better than the iterative one since it doesn't accumulate the prediction error but is computationally demanding in training [16], [17].

In this paper we suggest that a suitable combination of direct and iterative approaches would result with lower prediction errors when forecasting system states.

\section{THEORETICAL BACKGROUND}

\section{A. Gaussian Process Regression}

Our model is based on predicting the outputs using a Gaussian Process Regression (GPR) in an iterative manner. A Gaussian Process is a collection of random variables, any finite number of which have a joint Gaussian distribution [18]. In a GPR we assume that output is $y=f(x)+\varepsilon$, where it means that $y$ follows a latent function $f$ which is indexed by the observations $\mathrm{X}$ and corrupted by a Gaussian distributed noise $\varepsilon \sim \mathscr{N}\left(0, \sigma^{2}\right)$. Latent function $f$ is imposed by the prior:

$$
p(f \mid X)=\mathscr{N}(f \mid 0, K)
$$

where $K=k\left(x_{i}, x_{j}\right), \forall i, j \in\{1, \ldots, N\}$, is the Covariance Kernel of the process defined from the training data and $N$ is the length of training data. The Marginal likelihood of the process is defined as:

$$
p(f \mid X, \Theta)=\mathscr{N}\left(y \mid 0, K+\sigma^{2} \mathbb{I}\right)
$$

where $\Theta$ denotes the hyper-parameters that define the model and are estimated through the training procedure by maximising the log marginal likelihood:

$\log p(y \mid X)=-\frac{1}{2} y^{T}\left(K+\sigma^{2} \mathbb{I}\right)^{-1} y-\frac{1}{2} \log \left|K+\sigma^{2} \mathbb{I}\right|-\frac{N}{2} \log (2 \pi)$

The predictive distribution of mean $\mu\left(x^{*}\right)$ and variance $\sigma^{2}\left(x^{*}\right)$ of the new input $x^{*}$ is then calculated using:

$$
\begin{aligned}
\mu\left(x^{*}\right) & =k\left(x^{*}, x\right)\left(K+\sigma_{n}^{2} \mathbb{I}\right)^{-1} y \\
\sigma^{2}\left(x^{*}\right) & =k\left(x^{*}, x^{*}\right)-k\left(x^{*}, x\right)\left(K+\sigma_{n}^{2} \mathbb{I}\right)^{-1} k\left(x, x^{*}\right) \\
k\left(x^{*}, x\right) & =\left[C\left(x^{*}, x^{1}\right), \ldots, C\left(x^{*}, x^{N}\right)\right]
\end{aligned}
$$

where $\mathrm{C}$ are the covariance kernels relating the training and testing data.

\section{B. Covariance Kernels}

In the model, we are using the result over the summation of two different kernels:

1) Squared Exponential (SE) covariance function with Automatic Relevance Determination (ARD). This kernel helps in the modelling of the noise that arises through the data capturing process, as well as the vehicle dynamics since it assumes that "nearby inputs have high correlated outputs" [19].

$$
k_{S E}\left(x_{n}, x_{n^{\prime}}\right)=\sigma^{2} \exp \left(-\frac{\left(x_{n}-x_{n}^{\prime}\right)^{2}}{2 l_{n}^{2}}\right)
$$

2) Rational Quadratic (RQ) covariance function, also with ARD, helps to model sudden changes or step functions (eg. the user inputs).

$$
k_{R Q}\left(x_{n}, x_{n^{\prime}}\right)=\sigma^{2}\left(1+\frac{\left(x_{n}-x_{n}^{\prime}\right)^{2}}{2 a l_{n}^{2}}\right)^{-a}
$$

where :

- $\sigma^{2}$ is said to be the scale factor of each kernel; how far the data deviate from the mean.

- $l_{n}$ is the so called lengthscale of the kernel and determines how fast the data are changing. For each variable of the input $n$ there is a different lengthscale parameter; hence the term ARD. This shows how important that input is to the prediction of the particular output. The implication is that, non-important variables can be removed from the inputs so as to reduce the dimensionality of the model and decrease the computation time.

- $a$ is a weighting parameter for the RQ kernel.

For a detailed description of Gaussian Processes, further clarifications and references, the reader could follow [18]. For the implementation of the GPR algorithm we are using the Gaussian Process for Machine Learning (GPML) Matlab Code [20].

\section{THE EXPERIMENT PROCEDURE}

\section{A. The Simulator}

Data are collected through a racing car simulator software, rFactor, recommended for its excellent graphics and realistic vehicle dynamics by many users and racing teams. User experience and collection of truthful data is of primary importance, therefore we use demanding hardware specifications to provide the best graphic performance and avoid the generation of any game lag. As shown by Figure 2, we are using a custom set up of the Vision Racer VR3 seat with Logitech G27 Force Feedback Steering Wheel and a combination of three monitors to enhance the user's experience.

\section{B. The Experiment}

We collected data from 3 users, where each user was asked to drive a particular track twice for 15 laps with a 10 minute break in between. This approach gives us multiple varied data sets to train and test our individual user models. 


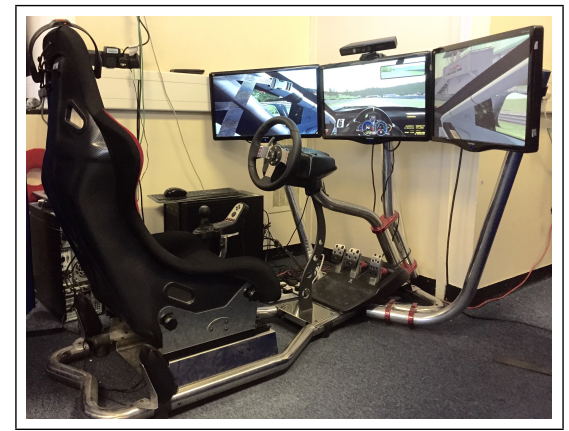

Fig. 2. Our custom car simulator setup enhances the user's experience for the data collection process.

Prior to starting the experiment, each user was instructed to pay special attention to:

- maintaining a comfortable driving speed

- avoiding accidents or crashes

- trying to achieve the best individual lap time without putting himself/herself into dangerous situations or getting out of the road boundaries

Each subject was given the opportunity to get to know the simulator through a single practice lap at the beginning of the experiment which is discarded from further analysis. Only one lap was allowed so as to avoid excessive familiarisation of the track's path.

\section{Car and Track Selection}

The car selected, from the ones available, was a sport version of Renault Megane. The requirements were to have a car that was easy to drive by an amateur driver, quite stable on the road, with a descent acceleration and top speed.

The experiments are loaded with The Limerock Park track, which is a short $(2.41 \mathrm{Km})$ but still challenging track with various types of segments (e.g. chicanes, straights, sharp turns), as seen in Figure 3.

\section{DATA ANALYSis}

The training of a GPR model requires the continuous inversion of the kernel matrix (K) of magnitude $n \times n$ to optimise the hyper-parameters. This is very computationally intensive (of order $O\left(n^{3}\right)$ ). User data is recorded at a frequency of $100 \mathrm{~Hz}$ so the amount of data gathered for even few laps makes it impossible to be used directly into a GP model. A solution is to segment the data and implement several user models, that are specific to the segmented path of the track. In this way the data sequences are shorter and also the model can be made simpler by omitting information regarding the curvature of the path.

\section{A. Track Segments}

Figure 3 shows the individual laps of 3 of our users in different colours. The rectangles indicate the 2 segments chosen, of different curvature and driving difficulty, for constructing the user models described in this paper.

The red rectangle has data from a short right turn followed by the previous long left turn: Region A. The car's speed was already reduced due to the difficulty of previous path, therefore results showed that the drivers were better prepared to carry out the manoeuvre. As seen in Figure 3, most of the user's paths created on that segment are dense and narrow. The blue rectangle encloses data from a long left turn which follows a long straight path: Region B. Results showed that non-professional drivers cannot keep track of their speed and most of the time they lose control of the car.

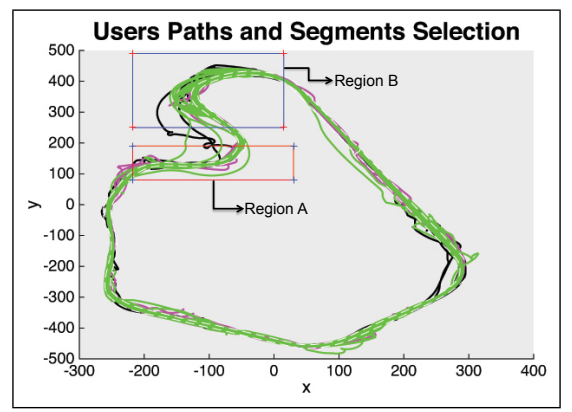

Fig. 3. Different Laps driven around the track by 3 users denoted by different colours. The driving skills of each user is dissimilar since they handle the path segments differently.

\section{B. Model Selection}

The algorithm makes inferences on how the car state and user behaviour (user inputs) change along the selected track segments. Therefore, sufficient information should be provided by the system for possible accurate predictions. Table I shows the 33 variables we use to implement the user model. These include forces acting on vehicle's centre of gravity, forces acting on each wheel independently - since this will result in a better real car state prediction - and the user input.

In a real world system, the environment state should also be embedded into the model as well. However, by using the same car, same paths and constant conditions (road type, weather, car damage, etc.) we can simplify and omit this information from the model. This is an attempt to model certain specific circumstances to test their applicability before moving on to a complete system.

TABLE I

VARIABLES OF THE USER MODEL IN THE GPR ALGORITHM.

\begin{tabular}{|l|l|}
\hline \multicolumn{2}{|c|}{ Variables } \\
\hline 1. Brake & 9. X/Y/Z Local Acceleration \\
\hline 2. Steering & 10. X/Y Local Velocity \\
\hline 3. Throttle & 11. X/Y Global Velocity \\
\hline 4. Gear & 12. Wheel's Rotation \\
\hline 5. Pitch & 13. Wheel's Lateral Force $(\mathrm{x} 4)$ \\
\hline 6. Roll & 14. Wheel's Suspension Deflection $(\mathrm{x} 4)$ \\
\hline 7. Yaw & 15. Wheel's Suspension Force $(\mathrm{x} 4)$ \\
\hline 8. Speed & 16. Engine RPM \\
\hline & 17. Time \\
\hline
\end{tabular}

\section{Model Training and Testing}

In this paper, we are trying to construct the user behaviour model of different segments with the assumption that the same trained model can be used for any path with similar settings (e.g. similar path, similar post and pre paths, type of road, same car, environment conditions, etc.). We collected 15 laps from each user in 2 different sessions, thus one of 
the sessions is used for training the model and the other for testing it. The user model consists of 33 Gaussian Processes predicting each of the variables in Table I. Another 2 GPs are used for the prediction of location change X-Y of the car. Our model is a form of an AutoRegressive (AR) model since output values depend on their previous inputs. It tries to map the inputs of normalised real values $(\tilde{X})$ from the variables in Table I of time $t$, to the change $(d x)$ of the same variables, at time $t+1$. As a result input is mapped to the output as follows:

$$
\tilde{X}_{t} \quad \rightarrow \quad d x=X_{t+1}-X_{t}
$$

Using this approach the model becomes more generic since different combination of inputs can be mapped to the same outputs. Different models are implemented, trained and compared using their RMSE scores along 1 and 2-second predictions. As shown by Figure 1, the User Model is implemented by training the appropriate combination of inputs and next state outputs using the GP algorithm. During testing process, the GPR uses the trained model with current state inputs at time, $t$, to provide the forthcoming state, $t+1$. Then $k$ forthcoming states are predicted by continuously fitting the result back to the prediction algorithm. Each forecast has an associate error therefore, forthcoming states propagate the errors from one prediction to the next.

We developed models that differ by two properties. One of the properties is the steps ahead prediction they are performing, so as to reduce the iterations of inputs fitted back to the GPR and therefore decrease the propagation of errors. This helps the prediction of slow varying variables since their value does not vary much between skipped time steps. The other property creates models with increased input dimensions of model state with past system states. Therefore, the state is comprised of the current values of the state as well as the past, instead of only the current system state, making a total of 66 input dimensions. However, this increases the computation time for training the algorithm but improves the prediction of variables that need more information than just the current state.

When naming the models the former property is defined by a number indicating the steps ahead to the default frequency $(100 \mathrm{~Hz})$ and the latter is defined by the words "Current" or "CAPI" (Current And Previous Inputs). Therefore if the model is called "GPR20Current" then is predicting 20 steps ahead $(20 / 100=0.2$ seconds $)$ on each iteration and the system state is composed of the current 33 variables.

In GPs the computation time depends on the size of the training data. Running Matlab on an Intel Core i7-2700K $(3.5 \mathrm{GHz})$ with $16 \mathrm{~GB}$ of RAM, the training time of the data from the 15 segment instances was varying between $60-120$ mins for every output variable, according to the properties of the model.

\section{RESULTS}

\section{A. The Testing Process}

For the purposes of this paper the testing is performed offline as we are interested in the applicability of the method and not its efficiency. As mentioned, every subject has 2 independent sets of data; one for training the model and one for testing it. Test data is made up of several instances (laps) of the selected trained path. From each path we can extract several current states to test with the model, using Equation (7). For example, if it took 10 seconds for the user to drive the selected segment and the model is trained with 20 steps ahead and we need a prediction time of 2 seconds, then we have 40 current states to test with the model. This number is also multiplied by the number of times the user performed the path, to find the total number of current states that the model can be tested with.

$$
C S=\frac{100}{S A} \times(\text { LPath }- \text { LPred })
$$

where:

- $C S=$ Available Number of Current States

- $S A=$ Steps Ahead

- LPath = Path Length $(s)$

- LPred $=$ Prediction Length $(s)$

For every prediction step of each model, the error is calculated as the absolute difference of the predicted value and the real value. Also, the error differences are accumulated from one step to the next where each step is averaged by the number of error accumulations. Lastly, all tested instances with their created current state error results are combined together using Root Mean Square Error (RMSE).

Apart from comparing each model, we also created a simple model, from each model type to use as a baseline. This model assumes a constant value along the prediction time. Following the same testing process, as expected, the simple models followed a similar narrow trend over the time of prediction. This is shown by the circle-dotted lines in Figures 4 and 5. This means that the different models can be compared to each other since varying the steps ahead does not seem to affect the error accumulation, which is less on lower frequencies, since there are fewer data points to compare.

\section{B. Trials}

Two different path segments have been modelled and tested offline using data obtained from 3 users of various driving skills. Figures 4 and 5 analyse data from a single subject since each model belongs to the particular driver from which the data was collected. Each user created in total 30 instances of each of the 2 segments shown in Section V-A.

1) Region A: Figure 4 shows the RMSE scores of prediction across time, for 3 different variables of the state (position $\mathrm{X}-\mathrm{Y}$ and speed), through the different models. All baseline models can be easily identified as they are grouped together and their RMSE lines linearly increase over time as expected. The steepness of the curves shows how fast that variable is changing across the region for the particular model. It can be noted that both dimensions of position have GP models underneath the baseline and their error is kept low along the 2 second prediction. Predictions of speed are all fairly close to the baseline, where as 6 of the GP models are better than the 


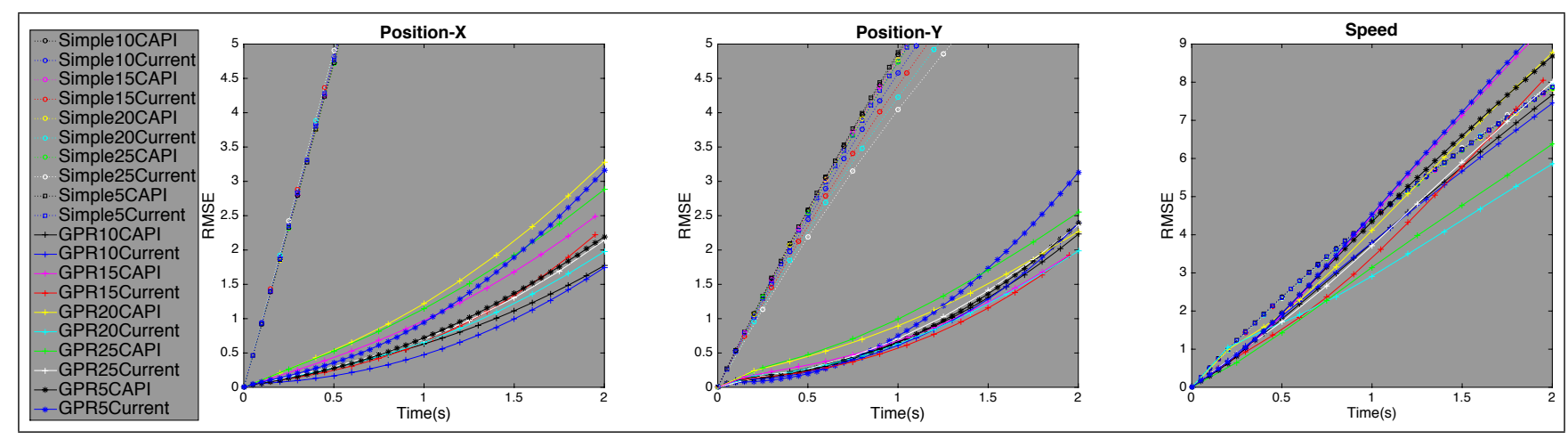

Fig. 4. Graphs show the 2-second RMSE of prediction to the real values of 3 state variables through different models, for Region A: Position in X-Y and Speed. Grouped dotted lines with circles are showing the RMSE from the Baseline (Constant) Models. All other plots show the RMSE of the GP-Models. Most of the GP models outperform the constant models on the prediction.

constant ("Simple"). We also noticed that larger steps ahead are more favourable and give lower errors. If lines are above their equivalent baselines it means their predictions are worse than the constant. This might be either because the training data are not informative enough, the model is not suitable or more information is needed in order to predict the output accurately.

2) Region B: The graphs on the first row of Figure 5 accumulate all errors from all test instances to show the general RMSE prediction, as on the previous section. As before, prediction of position for both axes is predicted better than the baseline by all the GP models. However, for speed the GP predictions vary and are spread around the baseline. This either means that the section is best modelled by a constant model (region B is a long curve driven with constant speed), GP cannot model the speed or the forecasted variables of the state introduce a lot of noise to the prediction.

In order to confirm the suitability of the models and find the most suitable one for each of the predicted variables, a more thorough analysis is needed. Instead of predicting all variables of the state and then feeding them back to the loop to find the next step, this time we will predict only one step of a particular variable and create the rest of the state from the offline data. As a result, only one variable will be predicted and carried along - the one in which we are interested - so there will be no aliasing from the inaccurate predictions from the other variables of the state.

This will give us the best possible prediction with a particular model for a certain variable. By repeating the testing for Region A and B with identical methods we found the results summarised in Table II. The table lists the best GP-Models for each variable and segment, with the RMSE scores over 1 and 2 second predictions. The best possible models are also shown with their scores, denoted by Region $\mathrm{A}^{\prime}$ and $\mathrm{B}^{\prime}$.

As expected, the RMSE of the predicted variables are reduced substantially for the best possible predictions A' and B'. Also, speed variable has shown improvement since more of the GP-models perform better than the baseline, as shown by the second row of plots on Figure 5 - especially models with lower than 10 steps ahead. Position seems to be modelled better by "Current" models and Speed by "CAPI". It is important to notice that, where there is a large difference in RMSE between the best possible prediction and current one, then this reveals that there is the potential of improvement. The next step of the research would be to remove the unsuitable inputs for each output and embed information that will reach the improvement scores.

TABLE II

Model SUITABILITY AND RMSE VALUES FOR 1 AND 2 SECONDS PREDICTIONS FOR REGIONS A AND B AND THEIR IMPROVEMENT.

\begin{tabular}{|c|c|c|c|}
\hline Variables & Best GP-Models & 1S RMSE & 2S RMSE \\
\hline \multicolumn{4}{|c|}{$\begin{array}{r}\text { Region A } \\
\end{array}$} \\
\hline Position-X & 10 Current/CAPI & 0.55 & 1.75 \\
\hline Position-Y & 15 Current/CAPI & 0.61 & 1.92 \\
\hline Speed & 20 Current / 25 CAPI & 3.04 & 6.12 \\
\hline \multicolumn{4}{|c|}{ Region $A^{\prime}$} \\
\hline Position-X' & 10 Current & 0.52 & 1.01 \\
\hline Position-Y' & 25 Current & 0.64 & 1.23 \\
\hline Speed' & 25 CAPI & 2.11 & 3.47 \\
\hline \multicolumn{4}{|c|}{ Region B } \\
\hline Position-X & 10 CAPI & 0.63 & 2.12 \\
\hline Position-Y & 10 CAPI & 0.63 & 2.21 \\
\hline Speed & 7 Current & 4.66 & 8.87 \\
\hline \multicolumn{4}{|c|}{ Region B' } \\
\hline Position-X' & 5 Current & 0.33 & 0.59 \\
\hline Position-Y' & 10 Current & 0.37 & 0.70 \\
\hline Speed' & 4 CAPI & 3.37 & 4.96 \\
\hline
\end{tabular}

\section{CONCLUSION}

In order to predict forthcoming car states using user models, we adopted a machine learning approach based on Gaussian Process Regression. Results after 1 and 2-second projections over different users revealed that the algorithm can retrieve the 2D position and speed of the vehicle with low projection error and chances of improvement.

An advantage of using a GP is that predictions are accompanied by a variance which argues on how certain the model is about the predictions, therefore a threshold of when the inference should be taken into account or not can be set. When new system states are observed then the model can update itself to include those states as well. Also, restrictions can be enforced on the predicted outputs when they are fitted back into the system, in order to improve their quality in case the algorithm deviates from the physical limits 


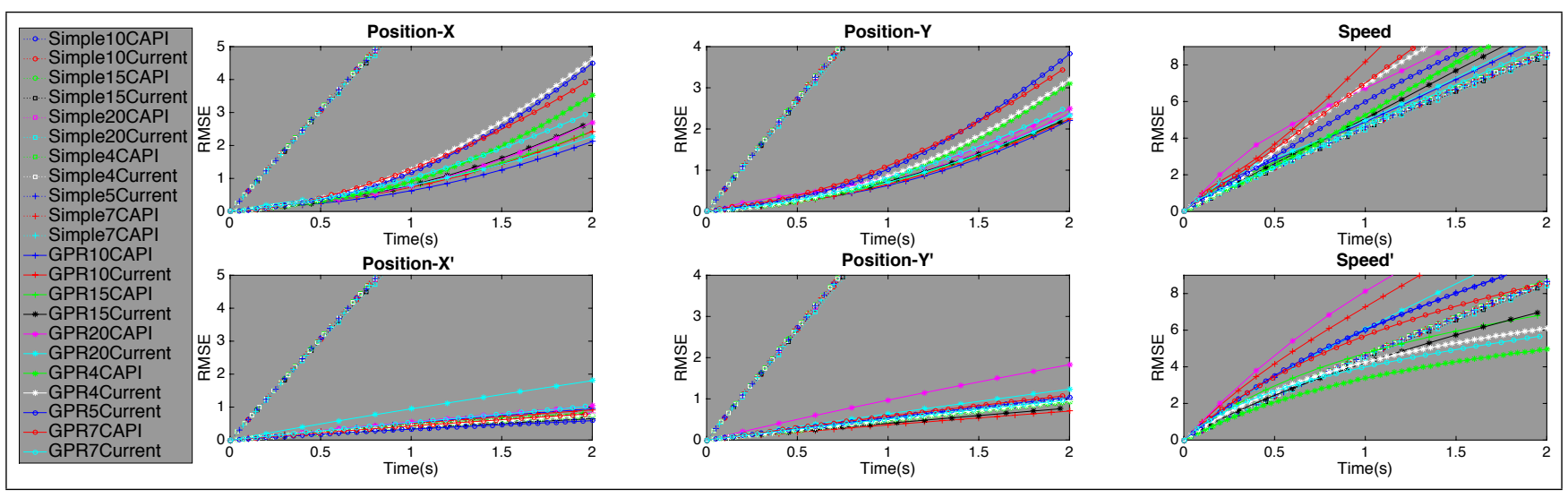

Fig. 5. First 3 graphs on the first row show the 2-second error accumulation for the prediction of 3 state variables through different models for Region B: Position in X-Y and Speed. Testing process keeps all Constant models together but it separates the GP-Models according to their RMSE scores. The 3 graphs on the second row show the 2 -second error accumulation as before with the difference that only 1 variable is being predicted; the one we are interested. For each variable this should be the best result possible that can be obtained from each of these models. This is because the input values that form the system state are taken from the real data (except the one we are predicting), for the whole prediction period. As expected the RMSE values are lower than the ones above.

(e.g. set minimum/maximum speed or acceleration of car). Another approach can be to blend multiple predictions from different models in order to determine a final value. We are also planning on testing a combination of different kernels to compare our results, as well as embedding vehicle motion models to the system.

By choosing a racing environment we are challenging the model to learn on the experience of a non-professional driver to cope with handling of a vehicle in a high speed driving environment. It can be argued that variables like position and speed are highly dependant on external parameters, other than the driver behaviour, and that they can be calculated using vehicle dynamic models. However, these kinds of models can only assume constant values for the variables needed to estimate the next states and this introduces unspecified errors. The predictions shown in this paper are entirely from a probabilistic approach trained through created states of a particular user with no prior knowledge of any kinematic formulae by the model, thus a variance is also predicted and errors are known.

In follow up work we aim to extend the system to include variables addressing additional environmental details such as pedestrians and other cars, increasing the applicability of our learning architecture to real world driving situations.

\section{REFERENCES}

[1] N. Oliver and A. P. Pentland, "Driver behavior recognition and prediction in a smartcar," in AeroSense 2000. International Society for Optics and Photonics, 2000, pp. 280-290.

[2] D. Mitrovic, "Reliable method for driving events recognition," Intelligent Transportation Systems, IEEE Transactions on, vol. 6, no. 2, pp. 198-205, 2005.

[3] Y. Liu, Y. Wang, W. Li, W. Xu, and J. Gui, "Improve driver performance by experience of driver cognitive behavior model's practice," in Intelligent Vehicles Symposium, 2009 IEEE, 2009, pp. 475-480.

[4] M. Panou, E. Bekiaris, and A. Touliou, "Adas module in driving simulation for training young drivers," in Intelligent Transportation Systems (ITSC), 2010 13th International IEEE Conference on. IEEE, 2010, pp. 1582-1587.

[5] H. Malik and A. Rakotonirainy, "The need of intelligent driver training systems for road safety," in Systems Engineering, 2008. ICSENG'08. 19th International Conference on. IEEE, 2008, pp. 183-188.
[6] T. Ranney, "Models of driving behavior: A review of their evolution," Accident Analysis \& Prevention, vol. 26, no. 6, pp. 733-750, 1994.

[7] T. Al-Shihabi and R. Mourant, "A framework for modeling humanlike driving behaviors for autonomous vehicles in driving simulators," in Proceedings of the fifth international conference on Autonomous agents. ACM, 2001, pp. 286-291.

[8] C. Miyajima, Y. Nishiwaki, K. Ozawa, T. Wakita, K. Itou, K. Takeda, and F. Itakura, "Driver modeling based on driving behavior and its evaluation in driver identification," Proceedings of the IEEE, vol. 95, no. 2, pp. 427-437, 2007.

[9] T. Wakita, K. Ozawa, C. Miyajima, K. Igarashi, K. Itou, K. Takeda, and F. Itakura, "Driver identification using driving behavior signals," in Intelligent Transportation Systems, 2005. Proceedings. 2005 IEEE, Sept 2005, pp. 396-401.

[10] E. Erzin, Y. Yemez, A. Tekalp, A. Ercil, H. Erdogan, and H. Abut, "Multimodal person recognition for human-vehicle interaction," Multimedia, IEEE, vol. 13, no. 2, pp. 18-31, 2006.

[11] A. Pentland and A. Liu, "Modeling and prediction of human behavior," Neural computation, vol. 11, no. 1, pp. 229-242, 1999.

[12] I. Dagli, M. Brost, and G. Breuel, "Action recognition and prediction for driver assistance systems using dynamic belief networks," Agent Technologies, Infrastructures, Tools, and Applications for E-Services, pp. 179-194, 2003.

[13] D. D. Salvucci, E. R. Boer, and A. Liu, "Toward an integrated model of driver behavior in cognitive architecture," Transportation Research Record: Journal of the Transportation Research Board, vol. 1779, no. 1, pp. 9-16, 2001.

[14] Y. Kishimoto and K. Oguri, "A modeling method for predicting driving behavior concerning with drivers past movements," in Vehicular Electronics and Safety, 2008. ICVES 2008. IEEE International Conference on. IEEE, 2008, pp. 132-136.

[15] J. Ko, D. J. Klein, D. Fox, and D. Haehnel, "Gaussian processes and reinforcement learning for identification and control of an autonomous blimp," in Robotics and Automation, 2007 IEEE International Conference on. IEEE, 2007, pp. 742-747.

[16] T. Hachino and V. Kadirkamanathan, "Time series forecasting using multiple gaussian process prior model," in Computational Intelligence and Data Mining, 2007. CIDM 2007. IEEE Symposium on. IEEE, 2007, pp. 604-609.

[17] W. Yan, H. Qiu, and Y. Xue, "Gaussian process for long-term timeseries forecasting," in Neural Networks, 2009. IJCNN 2009. International Joint Conference on. IEEE, 2009, pp. 3420-3427.

[18] C. E. Rasmussen, "Gaussian processes for machine learning," 2006.

[19] S. Brahim-Belhouari and A. Bermak, "Gaussian process for nonstationary time series prediction," Computational Statistics \& Data Analysis, vol. 47, no. 4, pp. 705-712, 2004.

[20] C. E. Rasmussen and H. Nickisch, "Gaussian processes for machine learning (gpml) toolbox," The Journal of Machine Learning Research, vol. 11, pp. 3011-3015, 2010. 